\title{
Surfaces
}

\section{Serge Gavronsky, Toward a New Poetics (University of California Press, 1994)}

\section{Katia Stockman}

Volume 5, 1995

URI : https://id.erudit.org/iderudit/1065009ar

DOI : https://doi.org/10.7202/1065009ar

Aller au sommaire du numéro

\section{Éditeur(s)}

Les Presses de l’Université de Montréal

\section{ISSN}

1188-2492 (imprimé)

1200-5320 (numérique)

Découvrir la revue

Citer ce compte rendu

Stockman, K. (1995). Compte rendu de [Serge Gavronsky, Toward a New Poetics (University of California Press, 1994)]. Surfaces, 5.

https://doi.org/10.7202/1065009ar 


\section{Compte rendu}

\section{Serge Gavronsky : Toward a New Poetics}

Katia Stockman

Université de Montréal

Département de Littérature comparée

stockmak@ere.umontreal.ca

Surfaces Vol.V.05 (v.1.0F - 22/12/1995)

Tout texte reste la propriété de son auteur. Néanmoins, SURFACES demande d'être citée à l'occasion de toute autre publication du texte en question.

ISSN: $1188-2492$

\section{Serge Gavronsky, Toward a New Poetics (University of California Press, 1994)}

Le livre de Serge Gavronsky aborde son sujet - dégager la poétique contemporaine française - à partir de plusieurs perspectives. Il s'agit en fait d'une triple approche: une première partie historique et critique, une série d'entretiens avec des écrivains français, suivis des traductions de quelques-uns de leurs textes, parfois inédits ou en cours d'élaboration. Ce livre permet avant tout d'approcher de près une certaine poétique contemporaine, puisqu'il nous donne l'opportunité d'en lire des extraits tout en faisant connaissance avec le point de vue des écrivains suivants, que Gavronsky a séparés en deux catégories: les poètes Michel Deguy, Joseph Guglielmi, Claude Royet-Journoud, Jacqueline Risset, Jean-Jacques Viton; les poètes-romanciers Jean Frémon, Emmanuel Hocquard, Marcelin Pleynet, Jacques Roubaud et les romanciers Liliane Giraudon, Leslie Kaplan et Maurice Roche.

Un Américain en France! Ce livre s'adresse en effet à des lecteurs américains; mais je le recommanderais surtout à des lecteurs français: je m'expliquerai.

Historique, entretiens et textes. Ces trois lieux où l'on peut se promener dans le livre pourraient paraître un peu éparpillés. Il y a de ça. Légèreté américaine ou capacité d'avoir une vision plus large? Rien à voir en tout cas 
avec les monographies sorbonicoles ou les anthologies arides et ardues de l'édition française!

L'idée est bonne en tout cas, elle permet d'empêcher la lassitude propre, quelquefois, à la lecture des textes contemporains. Le livre de Gavronsky est à la fois un bon outil de travail et de lecture universitaire, et un moyen de se familiariser avec ces vers disloqués, ces mots inventés, ces non-sens de l'écriture actuelle..., etc.

L'aperçu historique de la littérature et des courants intellectuels, de l'aprèsguerre jusqu'à nos jours, s'élabore à partir de l'état de la société française et de ses changements institutionnels. Au terme de son analyse, Gavronsky cherche à comprendre les enjeux de la spécificité d'une attitude d'écriture actuelle qu'il nomme "a new poetics":

By 'a new poetics' I mean to suggest not only a reconceptualization of the rules of poetry writing and the forms to be followed, but also the ideological justification for such changes. (p. 1)

Gavronsky identifie quelques données afin de comprendre le foisonnement littéraire dans la France des années 40 jusqu'aux années 60 , ces dernières constituant le point central de son analyse. Il nous rappelle à quel point l'idéologie dominante des années 60 était en rupture avec les valeurs traditionnelles; l'auteur illustre sa description de l'enthousiasme intellectuel de l'époque par des exemples types et bien connus (qu'il n'était pourtant pas inutile de rappeler) comme la polémique entre Barthes et Picard11. Il y a cependant à déplorer que Gavronsky prenne la revue Tel Quel comme phénomène et lieu principal de toutes les polémiques des années 60 . Certes, Tel Quel est surement l'une des revues les plus importantes de l'époque, parce qu'elle a brassé tous les thèmes révolutionnaires de la véritable brisure qui s'opérait alors dans la société française. Mais il me semble que l'avant-garde ne se résumait pas aux auteurs publiés par Tel Quel, comme le suggère l'auteur. La revue Change, dont il n'est pas fait mention par Serge Gavronsky me parait, par exemple, tout aussi importante (il est vrai que bien des auteurs publiaient dans les deux revues mentionnées: l'auteur aurait-il jugé bon d'éviter la redondance?). Une autre lacune, tout à fait délibérée, est dans le choix de ne pas parler des membres de l'Oulipo et de l'importance de ce groupe, au moins en ce qui concerne la réflexion sur les formes et les genres littéraires. Je dis lacune délibérée parce que Gavronsky explique lui-même ce choix (ou ce non choix) dans une note:

One may question why Oulipo (ouvroir de littérature potentielle) does not figure in my discussion. The reason is twofold. On the one hand, that group, founded in 1960 and composed of Georges Perec, Italo Calvino, Harry Matthews, Raymond Queneau, Jacques Roubaud, and others, remains a marginal experiment, the last formalist hurrah, as it were. On the others hand, Jacques Roubaud is one of the writers I interview. Through his observations, as well as Maurice Roche's reminiscences about Georges Perec, perhaps the most atractive figure in that group, we gain a sense of that movement as preoccupied with verbal and mathematical experimentation for its own sake. (note 1, p. 45) 
Pour moi ces arguments ne tiennent pas. Je suis tout d'abord loin de considérer l'Oulipo comme un phénomène marginal. Fondé par Queneau et François Le Lionnais - cet oubli montre que Gavronsky ne s'est décidément pas intéressé à ce groupe! - prend son sens dans son opposition à toute école littéraire et à la liberté qu'il veut manifester (liberté de parole et d'écriture enfin possible, même au sein d'un groupe d'avant-garde!). Le mieux est de laisser parler l'un de ses chefs de file actuel, je dis bien actuel, parce que l'Oulipo est loin d'être mort comme tend à l'affirmer Gavronskyㄹ:

C'est, bien évidemment, pour des raisons historiques, le groupe Surréaliste qui a servi de repoussoir à l'Oulipo. Raymond Queneau, en effet avait été surréaliste. Et, on le sait, il était sorti violemment de la secte bretonnienne. C'est indiscutablement en réfléchissant à ce malheureux exemple qu'il "inventa" les quelques règles, originales, suivantes (...):

- Le groupe de l'Oulipo n'est pas un groupe fermé. Il s'étend par cooptation à de nouveaux membres.

- Nul ne peut être exclu de l'Oulipo.

- En contrepartie (on n'a rien sans rien) nul ne peut démissionner de l'Oulipo, ni cesser d'en faire partie.

- Il s'ensuit que quiconque a été membre del'Oulipo le reste. Cela implique en particulier:

- Les morts font toujours partie de l'Oulipo.

- Pour corriger ce que la dernière règle a de trop contraignant, une exception à (d) a été prévue. On peut cesser de faire partie de l'Oulipo dans les conditions suivantes: en se suicidant, mais devant huissier, qui constatera que le suicide de l'oulipien considéré est, selon ses dernières volontés explicites, destiné à lui faire quitter l'Oulipo et à retrouver sa liberté de manœuvre pendant le reste de l'éternité $\underline{3}$.

Il me semble regrétable que Gavronsky accorde si peu d'importance à ce groupe, bien qu'il ait interviewé le même Jacques Roubaud, l'un de ses membres actuels les plus fameux!

$\mathrm{Au}$ risque de paraître trop traditionnelle (trop française?), j'aimerais souligner un autre problème dans le préliminaire historique. Gavronsky commence une première partie qui semble être le point de départ d'une étude chronologique et qu'il intitule "When Humanism Became a Dirty Word". Avec Césaire, Sartre, puis Ponge, il analyse la remise en question des 
modes de représentation de la tradition humaniste française, basée sur un passé colonialiste. Ces changements, explique l'auteur, prendront leur sens avec les écrivains de Tel Quel (encore eux!) dont l'esthétique sera fondée sur la linguistique. L'auteur revient alors aux surréalistes de l'entre-deuxguerres qui eux aussi critiquaient les valeurs bourgeoises..., etc. Bien, mais ensuite toute une partie est consacrée à Lacan et Derrida où l'écriture joue un rôle prédominant dans les controverses sur les relations entre le langage et les structures sociales. Rien de contestable dans tout cela, mais pourquoi revenir après à la Résistance française, à René Char, Louis Aragon: parler de ces poètes est tout à fait fondé, mais le lecteur finit par se perdre à l'intérieur de ces va-et-vient chronologiques ou, en tout cas, à ne plus pouvoir posséder une image claire des enjeux de chaque période. Un manque de clarté, donc, à force de vouloir donner trop de précisions.

Mais en brossant les contours de la société française et surtout de son système scolaire de l'après-guerre, Gavronsky donne les moyens aux lecteurs de réfléchir à partir des entretiens qu'il a réalisés avec les écrivains contemporains de son choix - je reviendrai plus loin sur ces choix. Une véritable approche critique manque cependant - mais ce n'était surement pas le propos de ce livre, déjà très dense (près de 400 pages). Il s'agit donc d'un bon survol historique s'il s'adresse à des lecteurs, américains ou autres, ayant une connaissance moyenne de la société française de l'après-guerre. Mais de nouveau, et au risque de me contredire, je dirais que ce survol est aussi très intéressant pour un lecteur français ou pour toute personne ayant fréquenté les institutions françaises de près.

Tout d'abord parce qu'il s'agit d'un vision Outre-Atlantique de la littérature française voire de la société française en général (qui se résume d'ailleurs à Paris dans la plupart des cas): il est toujours intéresant et stimulant (pour les Français plus que pour tout autre "peuple", suis-je tentée de dire!) de voir et d'essayer de comprendre l'image que l'on projette ailleurs...

Deuxième raison de conseiller ce livre à un Français: cette perspective américaine, qui base ses données sur une vision acérée des institutions françaises, apporte, à mon avis, un vent d'air frais - une bourasque ? - sur certaines approches "très françaises": je dirais traditionnelles, même en ce qui concerne la littérature contemporaine (quand elle est par hasard matière à discussion). L'analyse la plus intéressante de Gavronky est en effet pour moi celle qu'il fait du système scolaire d'avant mai 1968 en France; cette institution est, selon lui, à la base de la retenue littéraire et linguistique même lorsqu'il s'agit de décrire des faits relativement crus - des auteurs qu'il interroge dans son livre (tous avaient plus de vingt ans en 1968):

What, then, were these readings and exercices considered essential to a French education? Whether the student was asked to write an essay, recite a poem, or analyze a paragraph of prose, elegance and equilibrium were encouraged, to which might be added a sense of propriety, even in topics that engage the reader or writer in themes of violence. Reading Liliane Giraudon's short stories, one is immediately struck by the refinement and precision of language in its depiction of scenes of sexuality and violence. ( $p$. 22) 
Gavronsky, qui lui aussi a cotoyé de près l'école française, campe son analyse de l'influence scolaire sur l'écriture des écrivains français à partir de quatre hypothèses; il s'agit en fait des quatre grands principes de l'éducation française de l'avant 1968:

1. L'accent était mis sur la formation de gens lettrés, mais avec une grande insistance sur la forme: l'élève n'était pas censé, dit Gavronsky, avoir une attitude personnelle face aux mots; l'écriture était avant tout un exercice. Et l'auteur y ajoute aussi un autre type d'attachement à la forme:

Legible handwriting, correct spelling, neat presentation: these were the major concerns, when I went to primary school in France, when all I interviewed went to school. All of us were told to hand in our cahiers so that the teacher could grade them not for content but for how well the letters had been shaped, the paragraphs ordered. (p. 23)

2. L'explication de texte, exercice typiquement français, et d'ailleurs dénoncé dans les années 60 par Barthes comme une opération réductrice des forces du texte, rappelle Gavronsky.

3. La dissertation, au sujet imposé et à écrire avec clarté, économie de moyens et élégance.

4. Le thème et la version latine.

Quatre types de formation qui auraient profondément marqué les écrivains contemporains:

(...) children educated before 1968 in a French lycée, if they wished to succeed, had to adapt their individual capacities to the objective lessons established by the Ministry of Education. (p.24)

Étant moi-même une élève issue du système français, mais de l'après 1968, je dois avouer que la description de Gavronsky ne m'est pas étrangère; je reconnais dans ses grandes lignes l'expérience que j'ai vécue (subie?) en France - mes professeurs n'avaient-ils pas été formés avant 1968?-, avec une moins grande systématisation peut-être (mais comment puis-je réellement en juger?), grâce à quelques enseignants plus ouverts.

Finalement qu'apprend-on sur l'écriture de notre temps? Il est incontestable que les écrivains choisis par Gavronsky ont été fortement influencés par la littérature américaine de ce siècle: beaucoup d'entre eux sont des traducteurs de poètes ou de romanciers américains; Leslie Kaplan est ellemême américaine. En quoi consiste exactement cette influence? Gavronsky reste vague dans son analyse et l'on sent qu'il veut rester prudent à ce sujet: dégager une nouvelle poétique française sous influence américaine n'était pas son propos de départ, mais il est clair que son choix d'auteurs - tous attirés par les États Unis - et les questions qu'il pose à ce sujet tendent à montrer le rôle des interactions entre la France et l'Amérique du Nord. Ainsi, peu à peu, et pour chaque écrivain interrogé, on voit les contours de cette influence se dessiner: l'attitude d'ouverture des écrivains américains, 
leur capacité de créer de nouveaux mots, de nouvelles formes d'expression. Au moins pourrait-on localiser cette influence dans l'époustouflante liberté de la langue américaine qui dégage tous les enfants du système scolaire de l'avant 1968 des carcans rigides de la langue française. L'influence de l'américain va jusqu'aux philosophes français; je pense ici à Gilles Deleuze et Claire Parnet qui écrivent dans Dialogues $\underline{4}$ :

L'Américain-langue ne fonde sa prétention despotique officielle, sa prétention majoritaire à l'hégémonie, que sur son étonnante aptitude à se tordre, à se casser et à se mettre au service secret de minorités qui le travaillent du dedans, involontairement, officieusement, rongeant cette hégémonie au fur et à mesure qu'elle s'étend: l'envers du pouvoir. L'anglais a toujours été travaillé par toutes ces langues minoritaires, anglo-gaelic, angloirlandais, etc., qui sont autant de machines de guerre contre l'anglais. (...) Oh non, il ne s'agit pas de faire du patois ni de restaurer des dialectes, comme les romanciers paysans qui sont généralement gardiens de l'ordre établi. Il s'agit de faire bouger la langue, avec des mots de plus en plus sobres et une syntaxe de plus en plus fine. Il ne s'agit pas de parler une langue comme si on était un étranger, il s'agit d'être un étranger dans sa propre langue, au sens où l'américain est bien la langue des Noirs. Il y a une vocation de l'anglo-américain pour cela. Il faudrait opposer la façon par laquelle l'anglais et l'allemand forment les mots composés dont ces deux langues sont également riches. Mais l'allemand est hanté du primat de l'être, de la nostalgie de l'être, et fait tendre vers lui toutes les conjonctions dont il se sert pour fabriquer un mot composé: culte du Grund, de l'arbre et des racines, et du Dedans. Au contraire l'anglais fait des mots composés dont le seul lien est un ET sous-entendu, rapport avec le Dehors, culte de la route qui ne s'enfonce jamais, qui n'a pas de fondations, qui file à la surface, rhizome.

Cet engouement français pour la littérature américaine est surtout celui d'un groupe - pas d'une école - de plusieurs écrivains, parmi lesquels Gavronsky a puisé pour ses entretiens (j'aurais d'ailleurs à mentionner un oubli dans son choix: Dominique Fourcade qui n'hésite pas à mêler Anglais et Français dans sa poésie). Ainsi, rien d'étonnant que Jacques Roubaud ne lise pratiquement qu'en anglais et qu'il ait été l'un des premiers à publier, avec Michel Deguy une anthologie de la poésie américaine $\underline{5}$. De même, Emmanuel Hocquard a fondé un bureau sur l'atlantique et publié avec Claude Royet-Journoud deux anthologies $\underline{6}$. L'ouverture vers d'autres genres ou types d'écriture, la liberté syntaxique de la langue américaine est essentiellement ce qui ressort de tous ces entretiens d'écrivains qui ont en commun une attitude critique face aux institutions françaises et plus particulièrement face à l'institution "Histoire littéraire": je pense ici, par exemple, à Emmanuel Hocquard qui affirme préférer Chandler à Chateaubriand $\underline{7}$... 


\section{NOTES}

1Voir la réponse de Barthes à René Picard dans Critique et Vérité (Paris:

Seuil, 1966)

$\underline{2}$ «L'Oulipo a commencé en 1960 et il est toujours vivace. C'est un phénomème exceptionnel dans l'histoire de la littérature française» écrit Jacques Roubaud dans Poésie, etcetera: ménage (Paris: Stock, 1995): 197-198.

3Ilbid., p.199-200.

4(Paris: Flammarion, 1977): 72-73.

5Vingt poètes américains (Paris: Gallimard, 1980).

$\underline{6} 21+1$ poètes américains d'aujourd'hui (Monpellier: Delta, 1986) et 49+1 nouveaux poètes américains (Royaumont: Un bureau sur l'atlantique/Action poétique, 1991).

7Voir p. 232. 